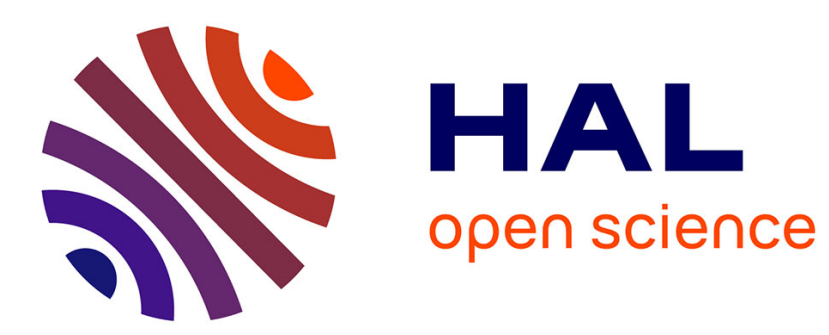

\title{
LA RECHERCHE SUR LA FUSION DANS LA COMMUNAUTÉ EUROPÉENNE
}

\author{
D. Palumbo
}

\section{To cite this version:}

D. Palumbo. LA RECHERCHE SUR LA FUSION DANS LA COMMUNAUTÉ EUROPÉENNE. Journal de Physique Colloques, 1977, 38 (C3), pp.C3-1-C3-7. 10.1051/jphyscol:1977301 . jpa00217089

\section{HAL Id: jpa-00217089 https://hal.science/jpa-00217089}

Submitted on 1 Jan 1977

HAL is a multi-disciplinary open access archive for the deposit and dissemination of scientific research documents, whether they are published or not. The documents may come from teaching and research institutions in France or abroad, or from public or private research centers.
L'archive ouverte pluridisciplinaire HAL, est destinée au dépôt et à la diffusion de documents scientifiques de niveau recherche, publiés ou non, émanant des établissements d'enseignement et de recherche français ou étrangers, des laboratoires publics ou privés. 


\title{
LA RECHERCHE SUR LA FUSION DANS LA COMMUNAUTÉ EUROPÉENNE
}

\author{
D. PALUMBO \\ Commission des Communautés Européennes
}

1. Pourquoi la Fusion en Europe ?. - Il n'est pas besoin de rappeler combien le problème de l'énergie est devenu pressant, sinon dramatique, pour l'Europe : les événements des dernières années l'ont suffisamment démontré.

En 1974, les pays de la Communauté Européenne ont consommé 10 millions de $\mathrm{GWh} /$ therm. d'énergie primaire; environ un quart de ces 10 millions ont été utilisés pour produire 1 million de GWh/électr. d'énergie électrique. Ceci a été obtenu en brûlant 1 milliard de tonnes équivalents de charbon (tec), en fait en majorité du pétrole $\left(0,8 \times 10^{9}\right.$ tec) en très grande partie acheté à l'extérieur et pour cela on a payé 50 milliards d'unités de compte (UC). Il nous appartient donc de chercher des sources d'énergie nouvelles permettant d'assurer l'indépendance économique de la Communauté. A l'échelle mondiale, les sources d'énergie à long terme qui s'offrent à nous sont les trois suivantes :

- les surrégénérateurs (fission),

- la fusion thermonucléaire contrôlée,

- l'énergie solaire.

On peut d'abord faire une comparaison entre les deux premières sources d'énergie constituées par l'énergie nucléaire (fission et fusion) d'une part, et l'énergie. solaire d'autre part. L'énergie solaire a le gros avantage de ne pas provoquer de nuisance et de constituer un réservoir inépuisable (tant que le soleil brûlera !). La physique en est connue et la solution de zones de captage de radiation (par miroirs, lentilles ou cellules photoélectriques) à la surface du sol (les fameuses fermes solaires) implique une technologie qui, bien que non encore développée, ne devrait pas présenter des difficultés insurmontables. Mais il convient de rappeler que les pays de la Communauté, vu leur densité de population et leur niveau industriel avancé, ont une consommation en énergie par $\mathrm{km}^{2}$ la plus élevée au monde (environ 4, fois celle des Etats-Unis). De plus, en ce qui concerne l'énergie solaire, la Communauté a deux ennemis : sa latitude asșez élevée et les nuages. D'une part, les surfaces nécessaires à la satisfaction des besoins s'élèveraient à environ $1 \%$ de la surface totale de la Communauté, ce qui poserait déjà des problèmes pratiques. D'autre part, il y aurait nécessité de stockage d'énergie non seulement entre le jour et la nuit, mais encore entre été et hiver, ce qui ne serait possible que par la voie chimique sans doute, sans parler des problèmes de transport de cette énergie des zones de captage (à faible densité de population) vers les villes.

Il apparaît donc que l'énergie nucléaire (fission et fusion) est la seule solution au problème de l'énergie en Europe. Si l'on compare les surrégénérateurs avec la fusion, les premiers, vu leur état de développement avancé, semblent présenter un avantage sur la fusion qui se trouve à un stade plus précoce. Leur commercialisation, à supposer que tous les problèmes soient résolus, ne saurait trop tarder.

Mais, à long terme, la fusion présente sans doute quelques avantages pour les raisons suivantes. Alors que les réserves en matières fissiles sont assez limitées en Europe, il n'en est pas de même du deutérium et du lithium nécessaires à la fusion. Le combustible deutérium se trouve en quantités pratiquement inépuisables dans les océans. Le lithium, lui, est nécessaire dans le réacteur à fusion pour produire du tritium. En effet, à cause de son haut rendement énergétique et de sa section efficace, seule la réaction deutérium-tritium doit être considérée dans le futur réacteur. Bien que le lithium ait été jusqu'à présent assez peu prospecté, il semble que nous en ayons des réserves relativement importantes. Des gisements de minerai avec une bonne teneur en lithium ont été découverts ces dernières années en différents endroits d'Europe. De bien plus grandes quantités de lithium se trouvent dans les couches plus profondes de la terre, mais en concentrations moins élevées. Enfin, il peut être extrait des océans, mais à un prix beaucoup plus élevé. Si la fusion était possible, on pourrait pallier les besoins actuellement non couverts par la production intérieure en utilisant 500 tonnes de lithium, ce qui coûterait environ $10 \mathrm{mil}-$ lions de UC (au lieu des 50 milliards de UC en 1974 mentionnés précédemment).

Un autre aspect est que les cendres résultant de la réaction de fusion sont stables ( $\mathrm{He} 4)$ : il n'en est pas de même des produits de la fission qui posent des problèmes d'évacuation. Il y a bien sûr production de tritium, qui est nocif; mais celui-ci reste dans l'en- 
ceinte même du réacteur et ne nécessite aucun transport. On peut donc dire en ce sens que la fusion est plus propre. D'autre part, la quantité de combustible présent dans la zone du réacteur est si modique que tout risque d'une fuite nucléaire dangereuse s'en trouve exclu. La chaleur nucléaire consécutive à un arrêt accidentel ne pose aucun problème sérieux. Pour ces raisons, le facteur sécurité est plus élevé dans la fusion. Enfin, la protection contre une utilisation abusive du système est facile à réaliser : le risque de sabotage est donc exclu tout comme la possibilité d'utilisation à des fins militaires, ce qui n'est pas le cas pour les surrégénérateurs.

Il faut cependant reconnaître un aspect du problème qui se révélera sans doute être ardu à résoudre : c'est celui de l'activation de l'enceinte du réacteur à fusion due au bombardement par des neutrons hautement énergétiques. Les contraintes qu'auront à subir les parois du réacteur sont énormes et ceci implique dès à présent, comme je le rappellerai plus loin, des études de matériaux très poussées. De ce point de vue, la fusion constitue un défi technologique.

Je n'ai pas parlé du charbon qui constitue une source d'énergie aux réserves non négligeables en Europe. Mais les frais d'extraction de celui-ci iront toujours en augmentant. Les frais d'extraction de l'équivalent énergétique en lithium sont dès à présent moins élevés : dans ce sens, il appartient de développer au plus tôt les recherches sur la fusion qui constituent - dans l'optique où le réacteur à fusion est possible et les résultats récents obtenus permettent de l'espérer un investissement rentable. Enfin, du fait que l'Europe dispose de matières premières très limitées et que le coût de sa main-d'œuvre rend ses industries de transformation moins compétitives sur le marché mondial, il lui appartient de former un personnel hautement qualifié afin de pouvoir, le jour venu, exporter son «know-how » vers des pays tiers.

\section{Organisation de la recherche sur la Fusion dans} la Communauté Européenne. La complexité des problèmes scientifiques et techniques qui requièrent d'énormes efforts et de longs délais, d'une part, l'ampleur des avantages potentiels, d'autre part, ont poussé nos pays à unir leurs efforts.

Un concours heureux de circonstances a permis à EURATOM de jouer en cela un rôle efficace : la fusion figurait en 1958 dans le programme initial de la CEEA; à ce moment, comme il est apparu évident lors de la conférence de Genève de la même année, une activité importante existait déjà aux Etats-Unis, au Royaume-Uni et en Union Soviétique. Dans les six pays qui à ce moment-là constituaient la Communauté Européenne, l'activité était à peine à ses débuts. Dans ces conditions, la Commission, plutôt que de créer son propre laboratoire au CCR, a préféré établir des Contrats d'Association avec les organisations nationales qui étaient actives dans ce secteur dans les deux buts : 1) de promouvoir l'action, 2) de la coordonner.

Cette politique a été poursuivie après l'entrée de la Grande-Bretagne, du Danemark et de l'Irlande dans la Communauté. Il y a une Association avec le Commissariat à l'Energie Atomique (CEA) en France avec des laboratoires à Fontenay-aux-Roses et à Grenoble. Une Association en Italie avec le CNEN et le CNR, laboratoire principal à Frascati et deux laboratoires encore à Padoue et Milan. Une Association avec le Max-Planck-Institut für Plasmaphysik avec son laboratoire à Garching près de Munich en Allemagne. Une Association avec la FOM en Hollande avec son laboratoire principal à Jutphaas près d'Utrecht. Une Association avec la Kernforschungsanlage en Allemagne, avec son laboratoire à Jülich. Une Association avec l'Etat belge concernant deux laboratoires à Bruxelles : l'Université Libre et l'Ecole Royale Militaire.

Ensuite se sont ajoutées l'Association avec l'UKAEA en Angleterre - avec son laboratoire de Culham - et l'Association avec le Danemark à Ris $\phi$. La dernière Association en date a été conclue avec la Suède, un pays ne faisant pas partie de la Communauté, en mai 1976 : elle concerne des laboratoires de Stockholm et Göteborg.

Toutes ces Associations sont gérées, en commun, par la Commission et l'Institution associées, et par un Comité de Gestion (CG). Cette gestion bilatérale a principalement un caractère administratif. En plus, toutes les Associations et la Commission ont constitué un Groupe de Liaison et un Comité des Directeurs :

- le premier, constitué en 1963, est chargé principalement de la préparation en commun des programmes et composé d'une trentaine de dirigeants scientifiques des Associations et de la Commission, qui en particulier décident des grosses expériences à lancer pour lesquelles une contribution de $45 \%$ est octroyée par la Commission ;

- le second, constitué en 1968, est composé des Directeurs des 8 Laboratoires Associés et du Programme de la Commission. Ce comité est particulièrement chargé de l'exécution des programmes et de l'échange de personnel, financé par la Commission, entre les Associations.

Ces deux organes sont assistés par des Groupes Consultatifs agissant sur les différents secteurs du programme (Tokamak, Stellarator, etc.) et par des Comités de coordination là où des actions sont menées en collaboration directe entre plusieurs laboratoires.

Enfin, le Comité Consultatif sur la Fusion (CCF), un organe à très haut niveau, a été créé début 1976. Il est composé de représentants de chaque Etat membre et Etat tiers, et de la Commission. Les avis du CCF, devant être une indication dans l'obtention d'une décision, sont transmis au Conseil et à la Commission. 
Ce système a fonctionné avec une certaine efficacité : un exemple de ce bon fonctionnement est la conception de la machine JET.

Quand les problèmes de la construction d'une grosse machine européenne se sont posés, il a été possible de parvenir à un accord complet sur le but et les dimensions d'une telle entreprise, qui maintenant a pris le nom de Joint European Torus (JET). Dans un délai très bref, il a été possible de rassembler un groupe international, auprès duquel les membres associés ont détaché des physiciens, et qui a produit, dans les délais prévus, un projet reconnu pour être le plus mûr parmi les projets similaires à l'échelle mondiale. Tous les jalons pour la phase de construction et d'opération de cette machine sont déjà posés. Un Comité de Surveillance est mis en place, qui devra assurer la surveillance et la réussite de l'entreprise, en étroite collaboration avec la Commission.

Tous ces comités, ces structures cités précédemment sont l'instrument qui permet à la Commission de poursuivre les deux buts déjà cités qu'elle s'est fixés : promouvoir l'action et la coordonner.

La Commission participe à cette activité de deux façons. D'abord, elle contribue par la mise à disposition de personnel scientifique et technique : un effectif d'environ 120 unités, JET mis à part. D'autre part, elle contribue par une participation financière différenciée, selon les objectifs poursuivis, d'après le schéma suivant :

- $25 \%$ des dépenses générales des Laboratoires Associés (salaires, frais de fonctionnement, etc...). Ce soutien uniforme à toutes les activités développées dans la Communauté dans ce domaine est l'instrument essentiel pour le maintien du caractère intégré $d u$ programme et la répartition des tâches entre les Laboratoires Associés ;

- $45 \%$ des coûts de construction des grands dispositifs expérimentaux dont l'usage est ouvert à tous les Associés. Le système du support préférentiel accordé aux actions prioritaires d'intérêt communautaire s'est révélé très stimulant et efficace ;

$-80 \%$ du coût total du projet JET. Cette participation relativement élevée de la Commission permettra aux Laboratoires Associés de poursuivre le reste $\mathrm{du}$ programme avec une concentration mais sans une réduction d'objectifs. D'autre part les Laboratoires Associés seront engagés aussi sur le plan financier dans la réalisation de ce grand projet commun.

Comme vous pouvez le remarquer, la contribution aux actions prioritaires est plus élevée que celle aux dépenses générales des Laboratoires Associés : ceci permet une stimulation entre les différents laboratoires et une coordination de leurs programmes. A titre d'exemple, le Tableau I vous indique la participation financière prévue de la Commission, suivant la ventilation indiquée précédemment, pour la période 1976-1980.
Le coût du programme, sans JET, s'élève à 415 MUC et la participation financière correspondante de la Commission sera de 124 MUC avec possibilité de révision selon les besoins ou les changements d'orientation au cours de cette période quinquennale. A titre d'exemple, il faut rappeler que pour la même période 1976-1980, les Etats-Unis estiment devoir dépenser près de 2 milliards de dollars. Même en incluant le projet JET, qui n'est pas encore approuvé, dans notre programme, l'on voit que la contribution des Etats-Unis représente environ 3 fois la nôtre. D'autre part, l'effort russe mesuré en scientifiques, puisque le mesurer en argent est difficile, représente également 3 fois le nôtre.

Laissez-moi enfin remarquer que le programme indiqué précédemment couvre la totalité de l'activité développée dans la Communauté dans le domaine de la fusion contrôlée. L'effort par habitant pour la recherche sur la fusion n'est donc environ que le quart de celui des Etats-Unis, là où le problème d'approvisionnement en énergie est moins crucial que chez nous.

\section{TABLEAU I}

\section{Programme pluriannuel proposé pour la période 1976-1980}

\begin{tabular}{|c|c|c|c|}
\hline & & \multicolumn{2}{|c|}{ Commission } \\
\hline Approuvé par le Conseil $\left({ }^{2}\right)$ & $\operatorname{MUC}\left({ }^{1}\right)$ & $\%$ & MUC \\
\hline Dépenses totales des Associations & 324 & 25 & 81 \\
\hline Nouveaux investissements & 87 & 45 & 39 \\
\hline Mobilité du personnel & 2 & 100 & 2 \\
\hline Direction et Administration & $\frac{2}{415}$ & 100 & $\frac{2}{124}$ \\
\hline Non encore approuvé & & & \\
\hline JET & $\frac{123}{538}$ & 80 & $\frac{98,4}{222,4}$ \\
\hline
\end{tabular}

( $\left.{ }^{1}\right) 1 \mathrm{MUC} \cong 1,2$ million de dollars USA.

$\left({ }^{2}\right)$ Décisions du 24 février et 18 novembre 1976.

3. Le but physique à atteindre et le programme actuel. - Le but physique à atteindre pour l'obtention du réacteur à fusion est déterminé par les deux impératifs suivants :

a) Un impératif physique, la faisabilité, d'après lequel le réacteur à fusion doit fournir au moins autant d'énergie qu'il n'en a été stocké dans la machine;

b) Un impératif économique, visant à une réduction maximum des coûts du réacteur et se traduisant par une optimisation nécessaire des paramètres de ce réacteur.

3.1 ImPÉRATIF PhySIQUe. - La difficulté inhérente à l'exploitation industrielle de la fusion réside dans le fait que la température de combustion de la réaction $\mathrm{D}+\mathrm{T}$ s'élève pratiquement à 100-200 millions de degrés $\mathrm{K}$.

Le problème est donc de chauffer le combustible 
à ces températures énormes et de le maintenir en condition de brûler, c'est-à-dire confiné dans un volume limité, pour une certaine durée.

Pour chauffer $1 \mathrm{~cm}^{3}$ de combustible à l'énorme température 'mentionnée, l'énergie requise $E_{\text {inp. }}$ sera proportionnelle à la quantité d'atomes contenus, donc :

$$
E_{\text {inp. }} \propto n .
$$

Une fois que le combustible est chauffé et brûlé, comme il s'agit de réactions binaires, la quantité de réactions par $\mathrm{cm}^{3}$ et par seconde est proportionnelle au carré de la densité. Donc, si on est en mesure de garder les conditions de combustion pendant un temps $t$, l'énergie produite est :

$$
E_{\text {out }} \propto n^{2} t .
$$

On aura donc un bilan d'énergie positif si

$$
E_{\text {out }}>E_{\text {inp. }}
$$

c'est-à-dire, dans le cas le plus favorable, si

$$
L_{\mathrm{D}-\mathrm{T}} \hat{=} n t>10^{14} \mathrm{~cm}^{-3} . \mathrm{s} .
$$

C'est le critère de Lawson illustré dans la figure 1.

$\mathrm{Si}$ on travaille à des densités très élevées on peut se contenter de temps très courts. Par exemple, à des densités environ 100 à 1000 fois celle de l'hydrogène solide, ce qu'on espère atteindre dans la fusion par laser, les temps requis seraient de l'ordre d'une nanoseconde et l'inertie de la matière suffirait à assurer ce temps de combustion (confinement inertiel). Mais si on utilise des combustibles de densité plus basse, ce

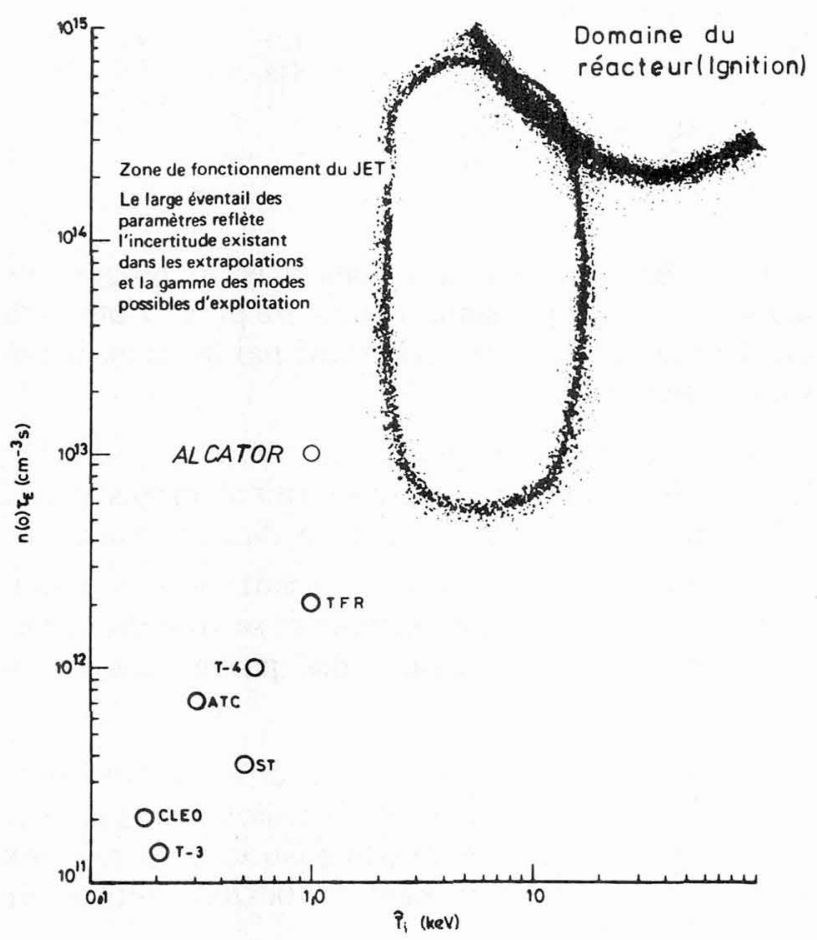

FIG. 1. qui semble plus simple, alors il faut des temps considérablement plus longs; par exemple avec les Tokamaks on espère avoir des densités $n$ de l'ordre de $10^{14}$ particules par $\mathrm{cm}^{3}$ et il faut donc des temps $t$ d'une seconde environ. Le recours à toute paroi métallique étant exclu, on se base sur le fait que les atomes sont totalement ionisés (et forment ce qu'on appelle un plasma), donc sensibles aux forces électromagnétiques : on peut avoir recours à des champs magnétiques. La plus grande partie de l'activité mondiale, et pratiquement toute celle d'EURATOM, est dirigée dans cette voie de recherche dont je vais parler maintenant (confinement magnétique).

Le principe le plus simple est de considérer un solénoïde cylindrique produisant un champ dont les lignes de force sont pratiquement axiales. Dans l'hypothèse la plus simple, l'équation magnétostatique :

$$
\nabla p=\frac{1}{c} j \times B
$$

indique que la pression $p$ est constante le long des lignes de force du champ magnétique. Donc, si l'on veut avoir un plasma confiné dans un volume limité, le champ magnétique doit lui aussi être confiné dans ce volume : ce qui nécessite la solution torique où le cylindre est replié sur lui-même. En fait, une composante azimutale, dite poloïdale, du champ est également nécessaire au confinement de façon que les lignes de force s'enroulent, en général sans fin, autour de l'anneau de plasma pour former des surfaces magnétiques où la pression du plasma est constante.

3.2 ImPÉRATIF ÉCONOMIQUE. - J'en viens maintenant à la valeur économique d'un système de confinement. On définit par bêta le rapport entre la pression du plasma qu'on peut confiner et la pression magnétique (proportionnelle au carré du champ) :

$$
\beta=16 \pi n k T / B^{2} \quad\left(T_{\mathrm{i}}=T_{\mathrm{e}}=T\right) .
$$

Ce coefficient $\beta$, qui ne peut dépasser un seuil critique défini pour chaque configuration, apparaît comme le facteur de qualité du système. En effet, la densité de puissance nucléaire (provenant des réactions de fusion) est proportionnelle au carré de la densité. D'autre part, pour des raisons mécaniques et économiques aussi, le champ magnétique ne peut dépasser certaines valeurs : on voit donc l'avantage économique d'obtenir un $\beta$ aussi élevé que possible. Je vais introduire maintenant la notion du $q$ qui peut être défini, pour les configurations axisymétriques, par le nombre de tours (pas forcément entier) qu'une ligne de force doit effectuer le long du tore afin de faire un tour autour de l'anneau de plasma. C'est au moyen de ces notions de $q$ et $\beta$ que je classifierai les différentes machines dont je vais parler maintenant.

Du fait que la solution des problèmes qui se posent 
en vue de la construction d'un réacteur à fusion demande un effort ininterrompu et que le projet de construction de grandes expériences s'étend sur de nombreuses années, les programmes de recherche sont quinquennaux, avec possibilité de révision en cours de programme. Le programme en cours couvre la période 1976-1980. Les investissements pour les actions prioritaires, en MUC, par ligne de recherche pendant le précédent et l'actuel programme vous permettent de voir quels genres d'activité ont été, et seront poursuivis dans l'avenir (Tableau II).

\section{TABLEAU II}

Ligne de recherche

Tokamaks $\left\{\begin{array}{l}\text { JET } \\ \text { autres }\end{array}\right.$

Stellarateurs

Conf. à $\beta$ élevé

Conf. ouvertes

Chauffage et injection

Technologie du réacteur

Syst. à très haute densité

Total sans JET

Investissements pour actions prioritaires (en MUC)

3.2.1 La ligne Tokamak $(q>1, \beta$ faible $)$. - Cette ligne de recherche était à l'origine confondue avec celle des Stellarateurs (pour former les configurations à faible $\beta$ ), l'importance des résultats obtenus par les Russes a montré la nécessité d'activer cette voie : c'est la raison pour laquelle fut introduit en 1971 le système d'actions prioritaires qui a permis à la Communauté de rattraper son retard dans ce domaine.

La composante poloïdale du champ, nécessaire au confinement du plasma, est produite en faisant circuler un courant électrique par induction dans l'anneau de plasma qui forme le secondaire d'un transformateur dont le primaire est constitué par des bobinages $\left(I_{\mathrm{p}} \neq 0\right)$. C'est le principe des pinches toroïdaux, et en particulier du Tokamak pour lequel le facteur de sécurité $q$ (pour des raisons de stabilité) est supérieur à 1 (de l'ordre de 2-3).

La construction de trois Tokamaks de dimension moyenne a été décidée en 1971, chacun ayant un but spécifique de recherche. Celle à Frascati du Tokamak FT permettra d'étudier l'extrapolation vers des champs magnétiques élevés (80-100 kGauss). La machine PULSATOR à Garching se distingue par ses bobines hélicoïdales qui augmentent la stabilité. Des résultats tout récents (décharge à haute densité) indiquent une densité de $1,7 \times 10^{14} \mathrm{~cm}^{-3}$, une température ionique de $0,34 \mathrm{keV}$ et un temps de confinement de l'énergie de $10 \mathrm{~ms}$. Je ne parlerai pas du Tokamak TFR construit à Fontenay qui fait l'objet d'un autre exposé à cette conférence. Permettez-moi seulement de men- tionner que cette machine, conçue pour l'étude des lois d'échelle, a été terminée en 1973 et a fourni les meilleurs résultats dans le monde à l'époque.

De petites machines sont également en opération : la machine ERASMUS à l'Ecole Royale Militaire à Bruxelles, caractérisée par un petit rapport d'aspect, la machine RINGBOOG à Jutphaas aux Pays-Bas permettant d'étudier la transition entre la couche de gaz neutre et un plasma totalement ionisé. A ne pas oublier la collaboration entre le M.I.T. aux EtatsUnis et certains laboratoires européens sur la machine ALCATOR. Le problème des impuretés est à l'étude dans deux machines spécialement construites à cet effet : la machine DITE à Culham en Angleterre utilise ce qu'on appelle un bundle divertor alors que la machine ASDEX à Garching, en cours de construction, aura un divertor magnétique axisymétrique.

Les problèmes restants, tels que ceux de la forme de la section du plasma, l'injection, la réalimentation seront traités dans des machines actuellement à l'étude. On envisage, par exemple, une version T2 améliorée du TFR à Fontenay, une machine DANTE à Ris $\varnothing$ au Danemark pour les Pellets. De son côté, la machine TEXTOR à Jülich en Allemagne permettra d'étudier l'interaction plasma-paroi.

Certaines machines sont spécialement conçues pour le problème de chauffage et j'aurai l'occasion d'y revenir à ce sujet.

Enfin, l'effort principal portera sur la construction et l'exploitation initiale d'une grande expérience Tokamak : le JET, qui fait l'objet d'un autre exposé à cette conférence. Je me bornerai à indiquer les objectifs scientifiques de cette expérience : produire un plasma à haute température ayant des paramètres proches de ceux nécessaires dans le réacteur, étudier le confinement dans le régime sans collisions et le comportement des particules alpha et, si possible, observer un début de chauffage nucléaire.

3.2.2 Les screw-pinches ( $q \sim 1, \beta$ fort). - Ces machines diffèrent peu du Tokamak, sauf que le champ magnétique est pulsé. Les recherches dans ce domaine se font sur la machine SPICA à Jutphaas $\left(\beta^{\mathrm{e}} \hat{=} 0,1-0,3\right)$.

Une catégorie de machines intermédiaires entre le Tokamak et le screw-pinch constitue les belt-pinches, caractérisée par une section allongée (grande ellipticité). Il convient de noter à Garching les machines BP II (pour la détermination d'un $\beta$ critique) et BP II a (pour le chauffage supplémentaire par ondes de choc et d'Alfven). Je mentionnerai encore les machines TENQ à Jülich et SP IV b à Jutphaas.

3.2.3 Les reversed pinches $(q<1, \beta$ fort $)$. Encore plus que les screw-pinches, ces machines se caractérisent par un fort $\beta$; d'où l'intérêt qu'elles suscitent du point de vue économique. Ces machines se caractérisent par un renversement de la direction du champ magnétique toroïdal, la composante poloïdale du champ est la plus importante et la théorie 
prévoit un $\beta$ assez fort pour des champs de quelques dizaines de kilogauss. Les recherches sont concentrées à Culham avec HBTX 1 et HBTX 2, et à Padoue avec les machines ÊTA-BÊTA (en collaboration avec Culham).

3.2.4 Les Stellarateurs à fort $\beta(\beta \sim 1)$. - Nous quittons maintenant les pinches toroiddaux pour aborder un autre système de confinement, celui des stellarateurs. Ici, la composante poloïdale du champ magnétique est produite par des bobines hélicoïdales et non plus par un courant toroïdal de plasma $I_{\mathrm{p}}$ induit $\left(I_{\mathrm{p}} \hat{=} 0\right)$. Les stellarateurs à fort $\beta$ ont la caractéristique supplémentaire que la compression et le chauffage du plasma sont obtenus par un champ magnétique variable dans le temps. Ces machines constituent, avec celles indiquées en 3.2 .2 et 3.2.3, l'ensemble des configurations à $\beta$ élevé indiquées dans le tableau II. Les études dans cette voie se font surtout à Garching avec ISAR T1 et où une nouvelle expérience (HBS-II) a été proposée.

3.2.5 Les Stellarateurs à faible bêta $(\beta \ll 1)$. Pendant le programme précédent, on a utilisé de nombreuses installations modestes : WENDELSTEIN IIa et IIb à Garching, PROTOCLEO à Culham, et on a construit des machines plus importantes : CLEO à Culham et WENDELSTEIN VIIa à Garching; cette dernière qui vient d'entrer en fonctionnement est le plus grand stellarateur actuellement existant. La construction de cette dernière machine a rencontré des difficultés techniques liées aux efforts mécaniques sur les bobinages hélicoïdaux. Il est à noter que les plasmas produits dans les Tokamaks et les stellarateurs ont des caractéristiques très proches, donc ces deux lignes de recherche peuvent s'épauler. Le stellarateur, d'ailleurs, connaît une certaine renaissance depuis la conférence de Berchtesgaden. Néanmoins, comme je l'ai déjà dit, le gros problème pour les stellarateurs de la dimension d'un réacteur sera celui des forces sur les bobines hélicoïdales.

3.2.6 Chauffage et injection. - En fait, cette ligne de recherche se retrouve dans toutes celles précédemment citées. Le chauffage ohmique n'étant pas suffisant, un chauffage additionnel s'impose donc. La voie du chauffage par ondes haute fréquence est surtout suivie aux laboratoires de Grenoble (CEA) et de l'Ecole Royale Militaire à Bruxelles. A cet effet on a construit la machine WEGA (en collaboration avec Garching) qui fonctionnera en stellarateur au début de 1977 et qui a été spécialement conçue pour étudier différentes méthodes de chauffage (chauffage à la fréquence hybride inférieure, synchrotron des ions...). D'autre part, le chauffage TTMP a été effectué sur le Tokamak PETULA à Grenoble également tandis que le chauffage haute-fréquence et au moyen de l'onde magneto-acoustique a été effectué sur le Tokamak ERASMUS à Bruxelles. Dans la machine TFR à Fontenay, on a commencé le chauffage synchrotron des ions.
Une autre voie qui semble très prometteuse est l'injection des neutres dont les recherches se font en collaboration étroite à Fontenay et Culham, et aussi à Garching. A cet effet, le développement de sources puissantes est en cours dans ces laboratoires. En particulier, le développement de la source et du système d'accélération pour la machine JET a commencé à Fontenay et Culham.

D'autres méthodes de chauffage sont à l'étude également, quoique à moins grande échelle, telles que la compression adiabatique, le chauffage par laser, le chauffage turbulent ou l'injection de granions.

3.2.7 Plasma à très haute densité. - L'activité coordonnée de plusieurs laboratoires sur le Plasma Focus (Frascati, Culham, Jülich) sera maintenue. Aucune nouvelle expérience importante n'est prévue avant que l'expérience de $1 \mathrm{MJ}$ actuellement en construction n'ait donné ses résultats.

Les expériences d'implosion des enceintes à Frascati ont été arrêtées au début du programme précédent, mais les contacts sont maintenus avec les travaux analogues poursuivis ailleurs.

Concernant le confinement inertiel (fusion par laser ou par faisceaux d'électrons relativistes), des activités théoriques et expérimentales sont déjà en cours dans quelques Laboratoires Associés. Une extension éventuelle de ces activités pourrait être envisagée selon des procédures flexibles, notamment en tenant compte de la situation particulière dans certains Etats membres dans lesquels ces activités sont développées dans le cadre de programmes classifiés.

Comme nous l'avons vu, les lignes de recherches déjà considérées sur lesquelles a été mis l'accent dans le présent programme sont les Tokamaks surtout, les systèmes à bêta élevé dans une moins grande mesure et le chauffage et l'injection. Mais la ligne de recherche qui a le plus grand développement, comparé au programme précédent, est la technologie du réacteur dont je vais maintenant parler.

4. Technologie et programme à long terme. - Les deux sujets, technologie et programme à long terme, sont extrêmement liés du fait que, dès qu'il y a une chance d'aboutir dans une voie (la faisabilité), alors se posent de nombreux autres problèmes à résoudre tels que ceux du champ magnétique, de la première paroi, de la chambre de recyclage du réacteur, du refroidissement, etc.

La première paroi du réacteur doit travailler dans des conditions extrêmement sévères. Elle subit le bombardement des radiations émises par le plasma et des particules qui, par diffusion ou par d'autres mécanismes, s'échappent du plasma ; elle est traversée par un flux important de neutrons et notamment par les neutrons de $14 \mathrm{MeV}$ produits par la réaction $\mathrm{D}-\mathrm{T}$; à travers des fenêtres qu'on doit pratiquer dans cette paroi, les cendres de la combustion, essentiellement de l'hélium et des atomes qui diffusent, doivent être évacuées, et ensuite du combustible frais 
doit être injecté. Cette paroi finalement doit être compatible avec la couche fertile de lithium ou contenant du lithium.

Cette couche de lithium est elle-même essentielle pour deux raisons :

- premièrement, parce que les neutrons y déposent leur énergie. Elle constitue ainsi la source de chaleur utilisable et elle doit travailler à une température suffisamment élevée (disons de 500 à $2000^{\circ} \mathrm{C}$ ) de façon à assurer la conversion de l'énergie thermique en énergie mécanique et ensuite électrique avec un bon rendement,

- deuxièmement, elle doit régénérer le tritium. L'épaisseur de cette couche de lithium doit être de l'ordre d'un mètre. Après la couche de lithium, il faut un écran destiné à absorber les neutrons résiduels et le rayonnement gamma produit dans la couche fertile. Finalement, il doit y avoir des bobines qui, pour des raisons économiques, devront probablement être supraconductrices et, dans ce cas, seront entourées par leur cryostat. Tout ceci sans parler des contrôles, du chauffage auxiliaire, etc.

Il s'agit d'une foule de problèmes technologiques extrêmement ardus dont l'analyse vient à peine de commencer. Ainsi, un réacteur à fusion doit être nécessairement grand et on pense qu'on pourra difficilement faire des unités économiques inférieures à quelques $\mathrm{GW}_{\text {électr. }}$, il sera donc très complexe et très coûteux.

Ceci demande de gros efforts et c'est pourquoi le budget pour la technologie du réacteur a été considérablement augmenté dans le présent programme (un facteur 8 par rapport au précédent).

En principe, une autre attitude serait possible : une attitude prudente qui consisterait à résoudre les problèmes physiques d'abord, c'est-à-dire à démontrer la faisabilité, sans se préoccuper des problèmes technologiques du futur réacteur. Cette attitude pourrait en fait s'avérer être coûteuse, car il ne sert à rien de démontrer cette faisabilité sur une machine qu'on ne peut pas utiliser comme réacteur. Nous avons opté pour la première attitude, plus risquante peut-être, qui consiste dès à présent à s'intéresser aux problèmes du réacteur, tout en tenant compte des interactions réciproques : c'est-à-dire faire de la physique en vue du réacteur. Dans cette voie, à mon avis la plus réaliste, une activité croissante est envisagée avec les objectifs suivants :

a) Acquérir l'expertise nécessaire pour la réalisation de JET et d'autres expériences nouvelles, dans des domaines tels que l'interaction plasma-paroi, les techniques du vide, la sélection et mise au point de matériaux, le projet et la construction de systèmes générateurs de champs magnétiques, les alimentations et les systèmes de contrôle;

b) Développer l'expertise nécessaire au projet et à la construction de la génération suivante d'expériences, dans des domaines tels que les bobines supraconductrices, le contrôle du plasma, l'activation des matériaux, la manipulation du tritium et la sécurité;

c) Poursuivre les études relatives à la faisabilité technologique et économique des réacteurs à fusion afin d'en identifier les aspects critiques. A l'appui de ces études on envisage d'effectuer quelques travaux limités d'ordre expérimental.

Ces trois lignes peuvent être couvertes par les activités développées dans le cadre des Laboratoires Associés d'une part et d'autre part par des souscontrats extérieurs placés par les partenaires associés sur des sujets bien définis et limités. Une coordination efficace et une interaction constante avec les laboratoires de physique du plasma sont requises. La seule vraie activité expérimentale actuellement existante concerne la physique des surfaces et l'interaction plasma-paroi auxquelles se consacre tout un département à Garching.

Il convient à ce sujet de noter que les Américains ont un programme à long terme dans lequel ils cherchent à déduire les voies à suivre, à la fois en physique et en technologie, en fonction des résultats obtenus en cours de programme.

Les services de l'agence américaine ERDA ont développé cinq stratégies (appelées logics) se distinguant les unes des autres par l'effort budgétaire annuel consacré à la fusion. La logic 1 caractérisée par un effort modéré, et constant dans le temps, n'aurait, selon l'opinion des Américains, aucune chance d'aboutir au réacteur. Dans la logic 2, bien que les fonds alloués chaque année augmentent, les progrès techniques seraient lents et le prototype du réacteur ne pourrait exister qu'au début du siècle prochain. La logic 3, appelée agressive, correspond à une augmentation du budget en fonction des besoins techniques supposés actuellement et conduirait à ce fonctionnement du prototype à la fin de ce siècle. La logic 4, dite accélérée, correspond à l'étude parallèle de nombreux problèmes et réduirait le délai de 5 ans environ. Enfin, la logic 5 correspond à l'effort maximum où les interactions entre les différentes actions engagées sont réduites, mais du fait des investissements énormes, le prototype serait attendu pour 1990. Une remarque intéressante sur ces différentes stratégies est que l'intégrale sur le coût, c'est-à-dire la somme totale des investissements jusqu'à l'avènement du prototype, a pratiquement la même valeur pour les logics $2,3,4$.

Dans l'état actuel des choses, l'agence ERDA semble être en faveur de la logic 3 (agressive) qui semble représenter une optimisation entre le désir d'aller de l'avant vers le but final et celui de minimiser le coût des investissements et les risques.

C'est sur cette optique des interactions réciproques entre les différentes lignes de recherches que nous avons basé notre programme dans la Communauté. 